\title{
Consequences of Magnetic Field Structure for Heat Transport in Magnetohydrodynamics
}

\author{
Shule Li \\ Adam Frank and Eric Blackman \\ Department of Physics and Astronomy, University of Rochester, Rochester, NY, 14627 \\ shuleli@pas.rochester. edu
}

Received — 


\begin{abstract}
Interfaces between hot and cold magnetized plasmas exist in various astrophysical contexts, for example where hot outflows impinge on an ambient interstellar medium (ISM). It is of interest to understand how the structure of the magnetic field spanning the interface affects the temporal evolution of the temperature gradient. Here we explore the relation between the magnetic field topology and the heat transfer rate by adding various fractions of tangled vs. ordered field across a hot-cold interface allow the system to evolve to a steady state. We find a simple mathematical relation for the rate of heat conduction as a function of the initial ratio of ordered to tangled field across the interface. We discuss potential implications for the astrophysical context of magnetized wind blown bubbles (WBB) around evolved stars.
\end{abstract}

Subject headings: magneto-hydrodynamics, planetary nebula, magnetic reconnection, wind blown bubbles, anisotropic heat conduction 


\section{Introduction}

Interfaces between hot and cold plasmas can occur in astrophysics where understanding the rate of thermal conduction may be an important part of the the astrophysical phenomenology. One example occurs in wind blown bubbles (WBB) of evolved stars where magnetized hot supersonic outflow shock heats the cooler ambient magnetized interstellar medium. For such WBB, there are examples where the presumed shock heated bubble is cooler than expected if only radiative cooling is considered (Zhekov et al. (2011)). A possible explanation is that heat loss through the interface of hot bubble into the cold shell via thermal conduction reduces the temperature of the hot bubble (Zhekov \& Park (1998), Zhekov \& Myasnikov (2000)). However the source of heat into the cold side of the interface will continuously evaporate material there and potentially induce interface instabilities and mass mixing (Stone \& Zweibel (2009)) that could tangle the magnetic field. Understanding the thermal conduction and its dependence on magnetic structure is important for determining the thermal properties of the plasma on either side of the interface.

A second example is the unexpected slow mass deposition rate of the cooling flows in some galaxy cores which might be inhibited by a restricted thermal conduction Rosner \& Tucker (1989), Balbus \& Reynolds (2008), Mikellides et al. (2011)). In the intracluster medium (ICM), the tangled magnetic field can potentially produce a strongly anistropic thermal conductivity that may significantly influence temperature and density profiles Chandran \& Maron (2004); Maron et al. (2004); Narayan \& Medvedev (2001), Mikellides et al. (2011)).

For the ISM and ICM, it is usually valid to assume that the electrons are totally inhibited from moving across field lines (McCourt et al (2011)), as the electron mean free path is much greater than the electron gyroradius. The magnetic field structure therefore 
plays a key role in controlling the rate of thermal conduction since electrons can move freely only along the field lines. The result is a strong thermal conductivity parallel to the field lines and a weak conductivity across the field lines.

The quantitative subtleties of how a complicated magnetic field structure affects thermal conduction for raises the open question of whether there is a simple measure of field tangling that allows a practical but reasonably accurate correction to the isotropic conduction coefficient for arbitrarily tangled fields. In this context, two classes of problems can be distinguished. The first is the conduction in a medium for which forced velocity flows drive turbulence, which in turn tangles the field into a statistically steady state turbulent spectrum (Tribble (1989), Tao (1995); Maron et al. (2004)). The second is the case in which the flow is laminar and the level of conduction inhibition is compared when the field starts from initial states of different levels of tangling subject to an imposed temperature difference across an interface. This second problem is the focus of our preset paper.

Using the ASTROBEAR magnetohydrodynamics code with anisotropic thermal conduction, we investigate the influence of initial magnetic structure on thermal conduction in an otherwise laminar flow. The key questions we address are: (1) does the interface become unstable? (2) how fast is the thermal conduction across the interface compared to the unmagnetized case?

We study these questions using different initial magnetic configurations imposed on a planar thermal interface to determine how the conduction depends on the amount of field tangling across the interface.

In section 2, we review the basic equations of MHD with anisotropic thermal conduction. In sections 3 and 4 we provide detailed description of the simualtion setup. In section 5 and 6 we present the simulation results and analyses. In section 7 , we discuss the simulation results in the context of the WBB cooling problem and the cooling flow problem 
in cores of galaxy clusters. The appendix provides more detailed information on the testing of the ASTROBEAR code.

\section{MHD Equations with Anisotropic Heat Conduction}

The MHD equations with anisotropic heat conduction that we will solve are given by:

$$
\begin{gathered}
\frac{\partial \rho}{\partial t}+\nabla \cdot(\rho \mathbf{v})=\mathbf{0} \\
\frac{\partial(\rho \mathbf{v})}{\partial t}+\nabla \cdot\left[\rho \mathbf{v} \mathbf{v}+\left(p+\frac{B^{2}}{8 \pi}\right) \mathbf{I}-\frac{\mathbf{B B}}{4 \pi}\right]=0, \\
\frac{\partial \mathbf{B}}{\partial t}+\nabla \times(\mathbf{v} \times \mathbf{B})=0, \\
\frac{\partial E}{\partial t}+\nabla \cdot\left[\mathbf{v}\left(E+p+\frac{B^{2}}{8 \pi}\right)-\frac{\mathbf{B}(\mathbf{B} \cdot \mathbf{v})}{8 \pi}\right]+\nabla \cdot Q=0,
\end{gathered}
$$

where $\rho, \mathbf{v}, \mathbf{B}$ and $p$ are the density, velocity, magnetic field, and pressure, and $E$ denotes the total energy given by

$$
E=\epsilon+p \frac{\mathbf{v} \cdot \mathbf{v}}{2}+\frac{\mathbf{B} \cdot \mathbf{B}}{8 \pi}
$$

where the internal energy $\epsilon$ is given by

$$
\epsilon=\frac{p}{\gamma-1}
$$

and $\gamma=5 / 3$. In our simulations, we will assume that the heat flux is confined to be parallel to the magnetic field lines. This assumption applies only when the ratio of electron gyro-radius to field gradient scales is small. Under this assumption, the heat flux parallel to field lines can be written as

$$
Q=-\kappa_{\|}(\nabla T)_{\|}
$$

where the subscript $\|$ indicates parallel to the magnetic field, and $\kappa_{\|}$is the classical Spitzer heat conductivity: $\kappa_{\|}=\kappa_{c} T^{2.5}$, with $\kappa_{c}=2 \times 10^{-18} \mathrm{~cm} \mathrm{sg}^{-1} K^{-2.5}$. We take $\kappa_{\|}$to be a constant throughout our simulations and so hereafter write it simply as $\kappa$. 
The ASTROBEAR code uses an operator splitting method to solve these MHD equations with heat conduction. The viscosity and resistivity are ignored in our calculation so the dissipation is numerical only. The ideal MHD equations are then solved with the MUSCL (Monotone Upstream-centered Schemes for Conservation Laws) primitive method with TVD (Total Variation Diminishing) preserving Runge-Kutta temporal interpolation. The result is then sent to the implicit linear solver utilizing the High Performance Preconditioners (Hypre) to solve the anisotropic heat conduction equation. The linear solver requires temporal sub-cycling technique to maintain its accuracy. The code runs in parallel with fixed grid domain.

\section{Problem Description and Analytical Model}

Our initial set up involves hot and cold regions separated by a thin planar interface. We study how the magnetic field configuration alters the heat transfer rate between the hot and cold regions in presence of anisotropic heat conduction. We study the problem in 2-D.

To guide subsequent interpretation of the results, we first compare two simple but illustrative limits of magnetic field orientation: (1) a uniform magnetic field aligned with the direction normal to the interface; (2) a uniform magnetic field perpendicular to the normal direction of the interface. In case (1), because the angle between the magnetic field and temperature gradient is everywhere zero, heat conduction across the interface is

expected to take on the Spitzer value associated with isotropic heat conduction. In case (2) however, the angle between the magnetic field and the temperature gradient is always $90^{\circ}$, so with our approximations, heat cannot flow across the interface.

We define a heat transfer efficiency $\zeta$ equal to the magnetic field-regulated heat transfer 
rate divided by the isotropic Spitzer rate, namely,

$$
\zeta=\frac{q}{q_{i}}
$$

where $q$ is defined as the amount of thermal energy transported through the interface per unit time. The average angle $\theta$ between the temperature gradient and the uniform magnetic field then plays an important role in determining $\zeta$. At $\theta=0, \zeta=1$. At $\theta=\pi / 2, \zeta=0$.

We now address the influence of both a mean field and a tangled field $\zeta$. Consider there to be a strongly tangled local field that has no mean value in the direction normal to the interface, i.e. $\mathbf{B}_{0, x}$ whose total magnitude is $B_{0}$, and a global magnetic field $\mathbf{B}_{d}$ aligned with the normal of the interface of magnitude $B_{d}$. If $B_{d} \gg B_{0}$, the magnetic field around the interface only slightly deviates from the normal direction and $\zeta$ should be close to 1 . If $B_{d} \ll B_{0}$, one would expect that the global energy transfer would be slow and $\zeta$ should be close to zero.

If $B_{d}$ and $B_{0}$ are comparable, we expect $0<\zeta<1$. We also expect $\zeta$ can change throughout the evolution if the strucuture of the magnetic field is modified by the dynamics of heat transfer. It is instructive to ask whether the feedback from the magnetic field structure evolution will amplify the heat transfer by creating more channels, or shut it down. The answer depends on the influence of magnetic reconnection, as we will see from the simulations. Only if magnetic reconnection acts to smooth out local small scale structures and link the initially isolated structures to the global mean field across the interface then we would expect the heat conductivity to increase.

\section{EDITOR: PLACE FIGURE[1] HERE.}

In what follows, we refer to the initial tangled field region as "the interaction region". Figure 1(a) shows a schematic of initial and hypothetical evolved steady state field 
configurations for such a tangled field set up. From the figure we can see that the initial field configuration forms a "wall" which restricts energy transfer across the two interaction region. However, if the subsequent evolution evolves to the steady state shown in (b), then expansion of the interaction region and magnetic reconnection has allowed the field to penetrate through the entire region. Thus the initial "wall" of tangle field wall is destroyed and thermal conduction will be less inhibited than initially. We will check how accurately this proposed picture of destruction of field wall is valid from analyzing our numerical simulations, and quantitatively discuss the effects on the energy transfer.

\section{Simulation Setup}

For our initial conditions, we set up an interface between hot and cold regions in mutual pressure equilibrium. The temperature distribution on the horizontal $(x)$ axis is given by:

$$
T(x)=T_{0}\left(1-x^{2}\right)^{0.4}
$$

in the region $0<x<1$ with $T_{0}=100$ in computational units. This temperature profile has a sharp gradient at $x=0$. The temperature distribution is plotted in Figure 2(a). The region $0.4<x<0.5$ is the interaction region we described in the previous section. At the two side boundaries, the temperature is set to be constant, and uniform across the regions of each respective side of the box connecting to that side of the interaction region. We are

primarily interested in the region of the box where the heat transfer occurs and noticeably evolves during the simulation run time. This means we will mainly focus on the interaction region. The horizontal length of the interaction region in the simulation domain is 0.1 in computational units. 
The thermal pressure is set to be in equilibrium over the entire box, that is

$$
P(x)=P_{0}
$$

with $P_{0}=100$. The density distribution is set up by the ideal gas law, namely:

$$
\rho(x)=\frac{P(x)}{T(x)}
$$

in computational units.

For the Spitzer diffusion coefficient, we assume the diffusion is linear as in Eq.(7), and use the approximation: $\kappa_{\|}=\kappa_{c} T_{m i d}^{2.5}$, where $\kappa_{c}$ is the classical conductivity, and $T_{m i d}$ is taken to be the middle value of temperature across the interface, about $0.5 T_{0}$.

We choose the initial field configuration:

$$
\begin{gathered}
B_{x}=B_{d}+B_{0} \sin (n \pi y / \lambda), \\
B_{y}=B_{0} \sin (n \pi x / \lambda)
\end{gathered}
$$

where $n$ and $\lambda$ are the mode number and wavelength of the tangled field respectively, $B_{0}=10^{-3}$ in computational units, and $B_{d}$ can assume various initial values that reflect the evolving global field as the result of reconnection. This initial field configuration is therefore one of a locally tangled field surrounding the interface with one measure of the tangle given by:

$$
R=B_{d} / B_{0}
$$

When $R=0$, there are only locally confined field lines, whereas $R=\infty$ indicates a straight horizontal field without any "tangling". As $R$ increases, the relative fraction of field energy corresponding to lines which penetrate through the interaction region increases. In our simulations, we consider cases with $R=0.0,0.2,0.4,0.6,1,2,4, \infty$. Figure 2(a), Figure 4(a) and Figure 5(a) show the magnetic field configuration for initial $R$ values of 0.0, $0.4,1.0$. 
We note that our MHD approximation a priori implies that the electron gydroradius is much smaller than the length scale of one grid cell. Thus the dissipation scale and all field gradient scales are larger than the electron gyro-radius by construction in our simulations.

We run simulations with typical resolution of 2048 cells on the horizontal axis in fixed grid mode. Runs with doubled resolution showed no significant differences compared to the standard resolution runs. We use fixed boundary conditions at the $x$ boundaries: the pressure, density and temperature at the two ends are fixed to their initial values, as is the magnetic field. We use periodic boundary conditions for the y-axis boundaries.

There are five parameters whose influence determine the simulation behavior and guide interpretation of results:

1. Plasma $\beta$. $\beta \equiv \frac{8 \pi P}{B^{2}}$ has little effect on diffusion because even with very high values of the plasma $\beta$ used in the simulation, we are still in the MHD regime and the gyro-radii of electrons are assumed small. Thus the direction of thermal conduction is not locally affected by $\beta$. It is possible that instabilities could arise in the low $\beta$ limit that affect pressure balance during the evolution of the simulations but that turns out not to be the case for the $\beta$ range of $10^{5} \sim 10^{8}$ that we use. The value of $\beta$ in this range does not exihbit any influence on the simulation result as indicated by our numerical experiment.

2. Initial Tangle measure $R=B_{d} / B_{0}$. If $R>>1$, the local small scale field can mostly be ignored and Spitzer thermal conductivity is expected, whereas if $R<<1$ a value much less than Spitzer is expected.

\section{Ratio of the diffusion time scale to the sound crossing time scale for one} grid cell:

$$
r=t_{d i f f} / t_{h y}=\frac{\rho C_{s} l}{\kappa_{\|}}
$$


where $\rho$ is the density, $l$ is the characteristic gradient length scale of temperature:

$l=\min \left(\frac{T}{|\nabla T|}\right)$ and $C_{s}$ is the sound speed. If $r<<1$, thermal diffusion would initially dominate and the pressure equilibrium would be broken by this fast energy transfer. If $r>>1$, then the pressure equilibrium would be well maintained throughout the entire evolution and the energy transfer may be viewed as a slow relaxation process. In our simulation, $r \approx 0.3$ initially, so that diffusion induces a pressure imbalance. Eventually, as the heat transport slows, the pressure equilibrium catches up and is maintained.

\section{Ratio between the temperature gradient scale length and the wavelength} of the tangled field: $h=2 \pi l / \lambda=k l$. If $h=0$ there is no tangled field, and no inhibition to heat transfer. As $h$ inreases, the field becomes more tangled, and the energy is harder to transfer. However, a large $h$ value may also result in increased magnetic reconnection, because the Lundquist number of field confined in a smaller region is larger, for the same field strength. Thus would then lower $h$.

5. Mean global energy transfer rate: $q=\delta E / t_{b a l}$, where $t_{b a l}$ is defined as the time needed for the hot region and cold region to reach a certain degree of temperature equilibrium by a transfer of heat energy $\delta E$ across the interface.

A mathematical expression for the heat transfer rate can be derived by considering a slab with a planar interface aligned with the $y$ direction at the middle of the interaction region $(x=0)$ with a tangled magnetic field, and an average temperature gradient aligned in the $x$ direction. Define the global temperature gradient as $|\nabla T|_{g}=\left(T_{\text {hot }}-T_{\text {cold }}\right) /\left(T_{0} L\right)$, where the subscripts "hot" and "cold" denote the characteristic temperatures of the hot and cold regions, $L$ is the width of the interaction region, and $T_{0}$ is a normalization factor which ensures $|\nabla T|_{g}$ has dimensions of inverse length. We can then integrate over the volume of the interaction region (and since there is no $z$-dependence, the essential content 
is an area integral) to obtain the effective heat flux through this region:

$$
\bar{q}=D|\nabla T|_{g} \int \frac{B_{d}}{|B|} d x d y,
$$

where $D$ is a constant that depends on neither the magnetic field nor the temperature distribution, $|B|$ is the local field strength. Notice that this expression is valid only when the magnetic field is varying at a length scale smaller than the interaction region length.

Using Eqs.(12), (13) and (14) in (16) and the approximation that the areal average in the interaction region $\left\langle\mathbf{B}_{0} \cdot \mathbf{B}_{d}\right\rangle \sim 0$ so that $\left\langle\left(\mathbf{B}_{0}+\mathbf{B}_{d}\right)^{2}\right\rangle \sim\left\langle B_{0}^{2}+B_{d}^{2}\right\rangle$, we obtain

$$
\bar{q} \approx D \frac{|\nabla T|_{g} R}{\sqrt{1+R^{2}}} .
$$

For the unmagnetized isotropic case, or for transfer with a field entirely aligned with the temperature gradient, we have instead

$$
\bar{q}_{i}=D|\nabla T|_{g} .
$$

Dividing Eq.(18) by Eq.(19), we obtain an appoximation for the heat transfer efficiency over the interaction region:

$$
\zeta=\frac{R}{\sqrt{1+R^{2}}} .
$$

If the initial temperature profiles are identical for different field configurations, this formula can then be used to estimate the expected energy tranfer rate from situations with various field configuration. By normalizing the heat transfer rate to that of the isotropic heat conduction case, we obtain the heat transfer efficiency $\zeta$. The accuracy of Eq.(19) can be tested by plotting the heat transfer efficiency obtained from the simulations against measured values of $R$.

If magnetic reconnection occurs during the time evolution of the heat transfer process, then conduction channels can open up and the energy exchange can be accelerated. We 
would then expect the actual curve of $\zeta$ vs $R$ to evolve to be higher than the value Eq.(19) predicts in situations with low $R$ values. Meanwhile, for high $R$, the analytical prediction and the real physical outcome should both approach the horizontal line $\zeta=1$, which denotes conductive efficiency consistent with the unmagnetized case. We emphasize that $R$ as used in this paper is always calculated with the the initial values of the magnetic field, not time evolved values, and that Eq.(19) is valid when estimating a cold to hot interface with initial tangle measure as the ratio of initial global straight field to initially local tangled field. To follow a measure of the tangle that evolves with time, a generalized tangle measure should be calculated in a more sophisticated manner and the integral form (Eq.(16)) should be applied.

\section{Simulation Results}

We choose initial conditions with values $R=0.0,0.2,0.4,0.6,1.0,2.0,4.0$ to run the simulations. The simulation run time is taken to be 1.2 (which corresponds to 12,000 years in real units for WBB. The initial cuts of temperature and magnetic field lines for $R=0.0,0.4,1.0$ are shown in Figure 2(a), Figure 4(a) and Figure 5(a) respectively. Figure 3(a) shows the initial cut of the density distribution in the $R=0.0$ run. We also run simulations with purely horizontal magnetic field lines, equivalent to the $R=\infty$ case, and runs with purely vertical field lines. Frames (b) to (d) in Figure 2 to Figure 5 are from the late stages of the evolution, and the final frames always display the steady state of the runs. A steady state is facilitated by the fact that the boundaries are kept at a fixed temperature throughout the simulations.

EDITOR: PLACE FIGURE 2 HERE. 
In Figure 6, we plot the mean cuts of the temperature $T_{c}$, obtained by averaging the temperature along $y$ axis, against the $x$ position for selected evolution times. Since the anisotropic heat conduction is initially faster than the pressure equilibration rate, the energy distribution around the temperature interface change rapidly until about $t=0.4$. This energy transfer is mostly confined to the interaction region for the low $R_{0}$ runs, since in these cases only a few field lines can penetrate into the entire interaction region.

EDITOR: PLACE FIGURE 3 HERE.

EDITOR: PLACE FIGURE 4 HERE.

\section{EDITOR: PLACE FIGURE 5 HERE.}

During the initial heat exchange phase, the thermal energy and density quickly

redistribute in the interaction region. As seen in Figure 2(b), islands at $x=0.48$ are formed by material bounded by the magnetic field lines, since the field orientation blocks heat exchange with the surroundings. Around $x=0.4$, there are also cavities formed where the thermal energy is inhibitted from flowing. The magnetic field lines, which form complete sets of loops in the $R=0.0$ case, begin to distort. It can be observed that the field lines are more strongly distorted in the low density part of the interaction region than in the high density part. This occurs because velocity gradients are driven by the early rapid redistribution of heat (pressure) by conduction.

At time $t=0.4$ (see Figure 2(b)), the field lines surrounding the cavities at $x=0.4$ reconnect, making thermal exchange possible. During the evolution, field lines begin to link the interaction region to the hot material on the left. This phenomenon is most apparent 
in Figure 2(d), which marks the final state of the thermal energy exchange. We also see that there is little difference between Figure 2(c) and Figure 2(d), because at late stage of the process, the thermal diffusion gradually slows so that the magnetic field configuration approaches a steady state.

By comparing Figure 6(c) with Figure 6(d), we see that the mean cuts of temperature show little difference for all values of $R$. The mean cuts of temperature $T_{c}$ exhibit a jump in the region of $x=0.35 \sim 0.5$, but are relatively smooth on either side of this region. This shows that even though the tangled field "wall" has been broken and allows channels of thermal conduction through it, the temperature profiles is not as smooth as in the purely straight field case.

\section{EDITOR: PLACE FIGURE 6 HERE.}

For the cases of $R=0.4$, there are field lines which penetrate the entire interaction region from the start. By observing the evolution of the magnetic field lines at about $x=0.38$, we see that magnetic reconnection is still happening, and causes the field loops

to merge. The observed behavior resembles the process displayed by Figure 1. When $R=1$, there are hardly any temperature islands that bounded by magnetic field loops. The evolution of the field lines shows less dramatic reconnection and evolve in what appears as more gentle straightening.

\section{Discussion}

EDITOR: PLACE FIGURE 7 HERE.

We begin our analysis with the evolution of the heat flux. The average heat flux per computation cell for different values of $R$ is plotted as a function of time in Figure 7 (a). 
Note that in the vertical field case $\left(\mathbf{B}=B_{y} \hat{y}\right)$ the heat flux remains zero as field entirely inhibits electron motion across the interface. For cases with $R>1$, the heat flux decreases throughout the evolution. Recall that $R>1$ implies cases where the "tangled" portion of the field is relatively weak and heat is quickly transported from one side of the interface to the other. Thus the trend we see for $R>1$ occurs as the temperature distribution approaches its equilibrium value. For lower $R$ values, especially those of $R<0.5$, an initial phase of heat flux amplification is observed as magnetic reconnection in the early evolution opens up channels for heat to transfer from hot to cold regions. At the late stage of the evolution when reconnection has established pathways from deeper within the hot region to deeper within the cold region temperature equilibration dominates leading to a decreasing heat flux phase as observed in the $R>1$ cases. Note that the similarity between the $R>2$ cases and the $R=\infty$ case is predicted by Eq.(19): as the global field comes to dominate, the heat flux inhibition imposed by anisotropic heat conduction in the local tangled field can be ignored.

\section{EDITOR: PLACE FIGURE 8 HERE.}

In order to understand the influence of magnetic reconnection on heat transfer rates we compare simulations with different filling fractions of the tangled field. Two cases are shown in Figure 8(a): (1) a temperature interface with a "volume filling" tangled field and (2) temperature interface with the tangled field filling only the region surrounding the interface. In case (2) the rest of the domain is filled with straight field lines connecting the hot and cold regions. From Figure $8(a)$ we see that case (1) shows much slower heat transfer rates compared to what is seen in case (2). This results because reconnected field lines in case (2) are linked to the globally imposed background field that in turn linking the hot and cold reservoirs. In case (1) reconnection only leads to larger field loops but cannot provide pathways between the reservoirs. The effect of different scale lengths on the evolving field 
loops is shown in Figure 8(b) in which we plot the result from three simulations wavelengths for the tangled field component (tangled field "loops"). Note that $\lambda$ is defined in Eq (12) and Eq.(13). We use a sequence of values for wavelength: $2 \lambda, \lambda$ and $\lambda / 2$. Figure $8(b)$ clearly shows that smaller field loop $\lambda$ leads to the largest average heat flux, since smaller scale loops will reconnect before large loops for a given magnetic resistivity. This result demonstrates the link between the number of reconnection sites of the field and heat flux.

We next analyze the temperature equilibration in detail. The averaged temperature difference across the interface is plotted in Figure 7(b). It shows the difference between the averaged temperature at the hot side and the cold side. One significant feature in Figure $7(\mathrm{~b})$ is that the temperature difference decreases to a steady value $T_{\text {end }}$ in all cases. This resembles percolation across a membrane which allows a density jump to happen when filtering two fluids. Figure $7(\mathrm{c})$ shows the distance required for the temperature to drop 80 percent at the interface. This distance characterizes the length of the interaction region. Except for the vertical field case where no heat transfer is allowed, the interface is expanding at different rates for different $R$ values. The expansion for all the cases of nonzero $R$ approaches a steady value which is also a characteristic feature of the temperature equilibration evolution.

We now analyze the modification of magnetic field configuration during the evolution. Throughout our simulations, the local magnetic field is initially a set of complete loops surrounding the interaction region. Once the energy transfer begins, the interaction region tends to expand as discussed previously. This expansion stretches the field lines on the $\mathrm{x}$ direction and distorts these circular loops, eventually inducing magnetic reconnection which oppens up channels connecting the hot and cold regions. From the current $J_{B}=|\nabla \times \mathbf{B}|$, we can get information on how tangled the field is. Figure 7(d) shows the evolution of the mean value of the strength of $\nabla \times \mathbf{B}$ in the interaction region. We observe that in the 
vertical and straight field case, $|\nabla \times \mathbf{B}|$ remains constant, but decreases to a fixed value for $R \geq 2$ cases. This means the field in high $R$ cases is straightened by the stretching of the interaction region as seen in Figure 7 (c). For the $R \leq 1$ cases, we see that $|\nabla \times \mathbf{B}|$ increases. This rise is due to magnetic energy brought in via the cold mass flow and the creation of fine field structures that amplify $J_{B}$ faster than dissipation caused by interface expansion.

\section{EDITOR: PLACE FIGURE 9 HERE.}

The local field distortion can be clearly demonstrated by studying the energy evolution of magnetic energy stored in different field components. In Figure 9(a), we plot the evolution of mean magnetic energy stored in the vertical field $\bar{B}_{y}^{2} / 2$, compared with $\bar{B}_{x}^{2} / 2$. We note that the latter includes only the fluctuating contribution to the energy in the $\mathrm{x}$ field - that is, the contribution to the horizontal field that does not come from the global mean $x$ component.

From Figure 9(a), we observe that the $B_{y}^{2}$ energy decreases while the $B_{x}^{2}$ energy either increases or remains the same for all cases. The magnetic energy evolution can thus be viewed as a conversion of vertical field to horizontal field. This conversion need not conserve the total magnetic energy of the local tangled field because of magnetic reconnection and because material advecting magnetic field can flow in and out of the interaction region. By comparison, in the $R>1$ cases, the thermal energy and local magnetic energy can both decrease and add to the kinetic energy of the material surrounding the interface, because of the fast thermal diffusion enabled by the strong global field.

The distortion of the local field loops can also be demonstrated by plotting the mean eccentricity of the field loops. In Figure $9(\mathrm{~b})$, we plot the mean eccentricity evolution. For all cases, the mean eccentricity is zero initially because of the circular shape of the field loops. Later in the evolution, large $R$ cases tend to evolve into a state of large eccentricity 
in the steady state. This is caused by a rapid expansion of the interface induced by the strong global field. In short, large $R$ induces more distorted local field loops and less tangled total field due to fast interface expansion, while small $R$ values results in less eccentric local field loops but with more tangled total field and strong magnetic reconnection.

\section{EDITOR: PLACE FIGURE 10 HERE.}

To compute the estimated heat transfer rate in the simulation, we calculate the averaged slope of the curve plotted in Figure 7(b), and compare it to the analytic model in Section 4. Although the equilibration rate represented by the slope of the curves in Figure $7(\mathrm{~b})$ is changing throughout the evolution, an early phase of the evolution can be chosen when the field configuration has not been modified significantly for which we can then comptute the averaged heat transfer rate. By normalizing the resulting heat transfer rate to the isotropic value, we can determine the heat transfer efficiency for different magnetic structures. From Figure 10, we can see that the analytic prediction and the simulation results agree quite well except for the situation when $R$ is below 0.2 . The simulation result does not converge to point $(0,0)$ but ends at an intercept on the $y$ axis. This intercept indicates that even if there are initially negligibly few channels for energy transfer, the magnetic reconnection can open up channels and allow heat transfer. Eq.(19) is valid for predicting the cooling rate of the hot material throughout the early phase of the heat equilibration process. It also provides insight on the strength of the local field in the vicinity of the interface once we know the cooling rate and global magnetic field strength.

To summarize our results we find first that the average heat flux at the end of our simulations is lower than at the beginning for all $R$ values. Thus we see an approach to thermal equilibrum. In some cases we also see that the heat shows an initially increasing phase denoting a period of active magnetic reconnection. 
In the simulations we see the average temperature difference decreases to a constant value $T_{\text {end }}$ which is related to $R$. We also see the width of the initial interface expand to a fixed value during the simulation.

Analysis of the simulation behavior shows that $J_{B}$ is an accurate measure of structural change in the magnetic field. Current decreases to a constant value for large $R$ cases and increases to a constant value for small $R$ values.

Finally we have shown that Eq.(19) can be used to estimate the energy transfer rate for an initially complicated field structure by considering the relative strength of the local field and the global field. For those cases for which $R$ approaches 0, Eq.(19) becomes invalid since the energy transfer in is mainly induced by a feedback from the magnetic field reconnection. By comparing cases with different field loop length scales, we demonstrate that the smaller the field loop length scale, the faster the reconnection rate.

\section{Astrophysical Applications}

\section{EDITOR: PLACE TABLE 1 HERE.}

The issue of magnetized conduction fronts and their mediation of temperature distributions occurs in many astrophysical contexts. One long-standing problem that may involve anisotropic heat conduction are hot bubble temperatures in Wind Blown Bubbles (WBB). WBB's occur in a number of setting including the Planetary Nebula (PN), Luminious Blue Variables (LBVs) and environments of Wolf-Rayet stars. When a central source drives a fast wind $\left(V_{\text {wind }} \sim 500 \mathrm{~km} / \mathrm{s}\right)$ temperatures in the shocked wind material are expected to be of order $10^{7} \mathrm{~K}$, which is greater than $2 \mathrm{kev}$. The temperatures observed in many WBB hot bubbles via from $\mathrm{X}$ ray emission are, however in the range of 0.5 kev to 1 kev range. NGC 6888 is a particularly well known and well studied example for a WR star 
(Zhekov \& Park (2010)). For planetary nebulae, Chandra X-ray observations have found a number of WBB hot bubbles with temperatures lower than expected based on fast wind speeds (Montez et al. (2005), Kastner et al. (2008)). The role of wind properties and heat conduction in reducing hot bubble temperatures has been discussed by a number of authors (Steffen et al. (2008), Akashi et al. (2007), Stute \& Sahai (2007)). The role of magnetic fields and heat conduction was discussed in Soker (1994).

While our simulations herein were meant to be idealized experiments aimed at identifying basic principles of anisotropic heat conduction fronts, we can apply physical scales to the simulations in order to make contact with WBB evolution. Tab.1 shows the results of such scaling. Upon doing so, we infer that: (1) given field strengths expected for WBB's, heat conduction is likely to be strong enough to influence on the temperature of the expanding hot bubble and the cold shell bounding it. We also note that magnetic fields in WBB (for PN field strengths see Wouter et al. (2006)) are usually in the milli-Gauss range, and are relatively much stronger than the field strength that can be scaled to our simulations. Thus the magnetic field in realistic WBBs is highly likely to result in anisotropicity and regulate the behavior of heat conduction. Since the heat transfer does not directly depend on the magnetic $\beta$, we can thus apply our analysis to the WBB interface if we approximate the interface to be planar and stationary, which is reasonable as the radius of curvature of WBBs are much greater than the interface scale of relevance. We must also assume that the global magnetic field is primarily radial.

The computational parameters used in our simulations and the real physics parameters typical in a WBB are listed in the first two columns of Tab.1. We choose the domain length to be $0.025 p c$, which is about 1 percent of the radius of the actual WBB. Tab.1 shows that by choosing the proper scaling, our simulation fits well with the data observed in a typical WBB. Therefore, the conclusions we draw by analysing the simulation results and 
the analytical expressions, especially Eq.(19), can be helpful in analyzing WBB evolution.

\section{Conclusion}

We have investigated the problem of heat transfer in regions of initially arbitrarily tangled magnetic fields in laminar high $\beta$ MHD flows using simulation results of ASTROBEAR code with anisotropic heat conduction. Three conclusions stand out:

(1) Hot and cold regions initially separated by a tangled field region with locally confined field loops may still evolve to incur heat transfer. The local redistribution of fluid elements bend the field lines and lead to magnetic reconnection that can eventually connect the hot and cold regions on the two sides. (2) The temperature gradient through such a penetrated tangeld field region tends to reach a steady state that depends on the energy

difference between the hot and cold reservoirs on the two ends. (3) Eq.(19), a measure of the initial field tangle, is a good predictor of the ultimate heat transfer efficiencies across the interface for a wide range of $R$.

A basic limitation of our simulations is that they are 2-D. A 3-D version of this study would be of interest as the field would then have finite scales in the third dimension possibly allowing channels for heat transfer excluded in 2-D. We have also not considered the effects of cooling in our simulations.

Future directions of analysis could include a multi-mode study, which investigates the effect of the spatial spectrum of the magnetic field distribution on the heat transfer efficiency. When there are multiple modes or a spectrum is continuous, it would be useful to predict how the efficiency would depend on the spectrum. In this context, a more detailed comparison of heat transfer in initially laminar versus initially turbulent systems would be of interest. 


\section{9. acknowledgements}

Financial support for this project was provided by the Space Telescope Science Institute grants HST-AR-11251.01-A and HST-AR-12128.01-A; by the National Science Foundation under award AST-0807363 and NSF PHY0903797; by the Department of Energy under award de-sc0001063; and by Cornell University grant 41843-7012. We wish to thank Jonathan Carroll, Kris Yirak and Brandon Shroyer for useful discussions.

\section{Appendix: Code Test}

The MHD solver and the linear thermal diffusion solver are verified by well-known tests such as the field loop convection problem and the Guassian diffusion problem separately. As a comprehensive test that involves both MHD and thermal diffusion, we use the magneto-thermal instability (MTI) problem to test the accuracy of the ASTROBEAR code with anisotropic heat conduction (Parrish \& Stone (2005), Cunningham et al. (2009)). The problem involves setting up a 2-D temperature profile with uniform gravity pointing on the y direction. The domain is square with length of 0.1 in compuational units. The temperature and density profiles are:

$$
\begin{aligned}
& T=T_{0}\left(1-y / y_{0}\right) \\
& \rho=\rho_{0}\left(1-y / y_{0}\right)^{2}
\end{aligned}
$$

EDITOR: PLACE FIGURE 11 HERE.

EDITOR: PLACE FIGURE 12 HERE. 


\section{EDITOR: PLACE FIGURE 13 HERE.}

with $y_{0}=3$. The pressure profile is set up so that a hydrostatic balance may be achieved with uniform gravity with gravitational acceleration $g=1$ in computational units. We also set $T_{0}=1$ and $\rho_{0}=1$ in computational units. There is a uniform magnetic field on the $\mathrm{x}$ direction with field strength $B_{0}=1.0 \times 10^{-3}$ in computational units. The anisotropic heat conductivity is set to be $\kappa=1 \times 10^{-4}$ in computational units. We use the pressure equilibrium condition for the top and bottom boundaries, that is, the pressure in the ghost cells are set so that its gradient balances the gravitational force. On the $\mathrm{x}$ direction, we use the periodic boundary condition.

Initially, the domain is in pressure equilibrium. We then seed a small velocity perturbation:

$$
v_{\text {per }}=v_{0} \sin (n \pi x / \lambda)
$$

with $v_{0}=1 \times 10^{-6}$ and $\lambda=0.5$. This perturbation will cause the fluid elements to have a tiny oscillation on y axis as well as the field lines. Once the field lines are slightly bent, they open up channels for heat to transfer on the y direction thus allowing the heat on the lower half of the domain to flow to the upper half. It can be shown that this process has a positive feedback so that once the heat exchange happens, more channels will be openned up for heat conduction. Therefore this process forms an instability whose growth rate can be verified according to the linear instability growth theory. We use $\tau_{s}$ to denote the sound

crossing time for the initial state. Figure 11 shows the time evolution of the field lines at various stages in our MTI simulation.

We study the MTI growth rate by considering the acceleration of the fluid elements. The mean speed on the y direction for the fluid should follow the exponential growth: 


$$
v_{y}=v_{p e r} e^{\gamma t}
$$

where $v_{p e r}$ is the strength of the initial velocity perturbation applied, $\gamma$ denotes the growth rate in the linear regime. We obtain the growth rate $\gamma$ by plotting $\ln v_{y}$ against the evolution time and then measuring the local slope through a certain time span. The $\ln v_{y}$ vs $t$ curve is plotted in Figure 12 (a), which shows a nice linear relation. We plot the growth rate against evolution time. It should be stable around the theoretical value 0.4 initially and then decrease sharply due to the nonlinear effect. Figure $12(b)$. shows that the simulation meets our expectation fairly well.

We also look at the energy evolution in the linear regime. The mean kinetic energy should first stay stable and then enter into an exponential growing phase until it hits a cap at around $t=200$ which denotes the starting of the nonlinear phase. The evolution of magnetic energy should follow similar pattern as to the kinetic energy evolution, but lagged behind. In Figure 13, we plot the time evolution of the mean kinetic and magnetic energy evolutions. The results confirms the physical intuition quite well. 


\section{REFERENCES}

Akashi, M., Soker, N., Behar, E., \& Blondin, J. 2007, MNRAS, 375, 137

Balbus, S. A., \& Reynolds, C. S. 2008, ApJ, 681, 65

Chandran, B. D. G., \& Maron, J. L. 2004, ApJ, 602, 170

Cunningham A. J., Frank A., Varnière P., Mitran S., Jones T. W. 2009, ApJS, 182, 519

Kastner, J. H., Montez, R., Jr., Balick, B., \& De Marco, O. 2008, ApJ, 672, 957

Maron, J., Chandran, B. D., \& Blackman, E. 2004, Phys. Rev. Lett., 92, 045001

McCourt, M., Parrish, I. J., Sharma, P., Quataert, E. 2011, MNRAS, 413, 1295

Mikellides, I. G., Tassis, K., \& Yorke, H. W. 2011, MNRAS, 410, 2602

Montez, R., Jr., Kastner, J. H., De Marco, O., \& Soker, N. 2005, ApJ, 635, 381

Narayan, R., \& Medvedev, M. V. 2001, ApJ, 562, 129

Parrish,I.J.,\& Stone,J.M. 2005, ApJ, 633, 334

Rosner, R., \& Tucker, W. H. 1989, ApJ, 338, 761

Soker, N. 1994, ApJ, 107, 276

Steffen, M., Schonberner, D., and Warmuth, A. 2008, A\&A, 489, 173

Stone, J. M., \& Zweibel, E. G. 2009, ApJ, 696, 233

Stute, M., \& Sahai, R. 2006, ApJ, 651, 882

Tao, L. 1995, MNRAS, 275, 965

Tribble, P. C. 1989, MNRAS, 238, 1247 
Vlemmings, Wouter H. T., Diamond, P. J., Imai, Hiroshi 2006, Nature, 440, 58

Zhekov, S. A., \& Myasnikov, A. V. 1998, New A, 3, 57

Zhekov, S. A., \& Myasnikov, A. V. 2000, ApJ, 543, 53

Zhekov, S. A., \& Park Sangwook 2010, ApJ, 721, 518

Zhekov, S. A., \& Park Sangwook 2011, ApJ, 728, 135 

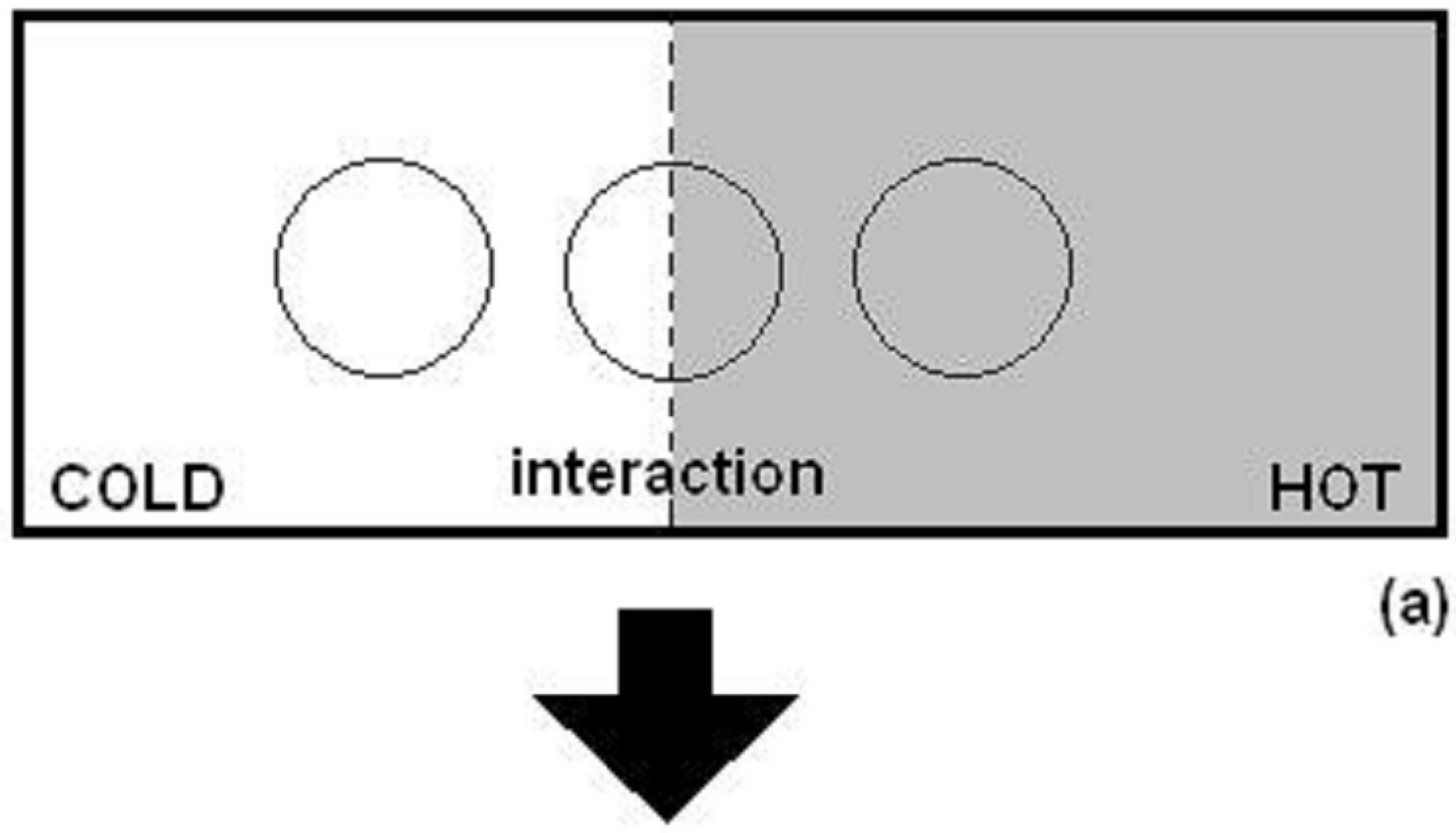

(a)

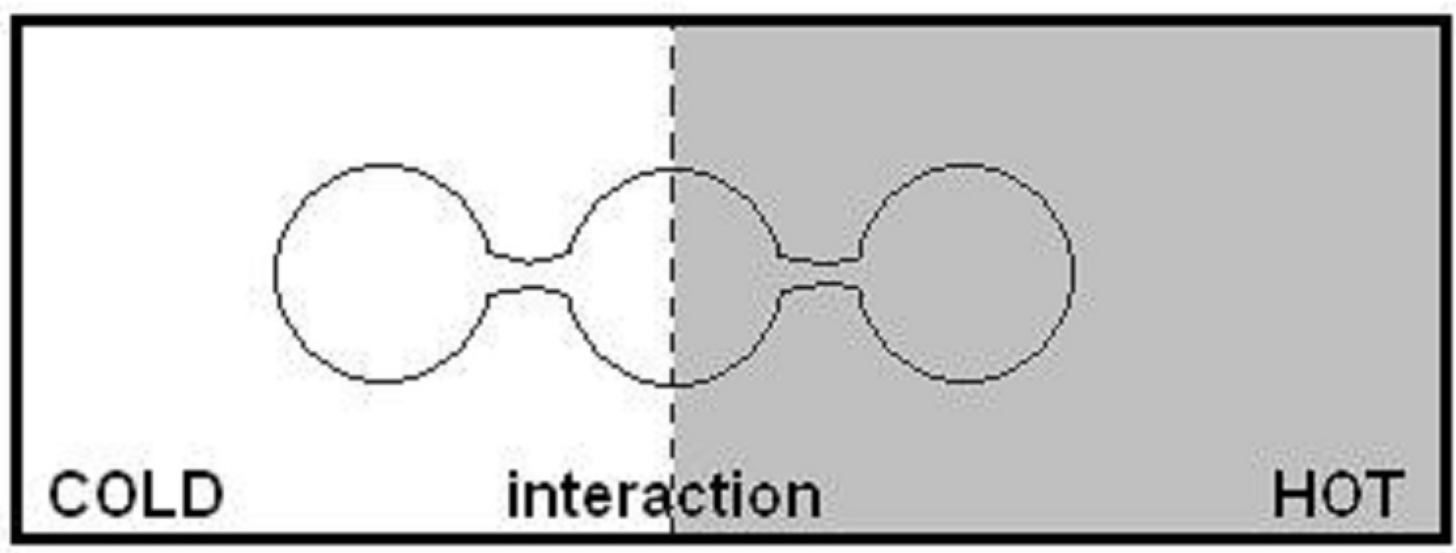

(b)

Fig. 1. - The initial and steady state field configuration. (a): the initial field forms complete loops that only allows heat transfer within the interaction region. (b): the steady state field reconnects itself so that it allows heat transfer between regions deeply into the hot and cold areas. 

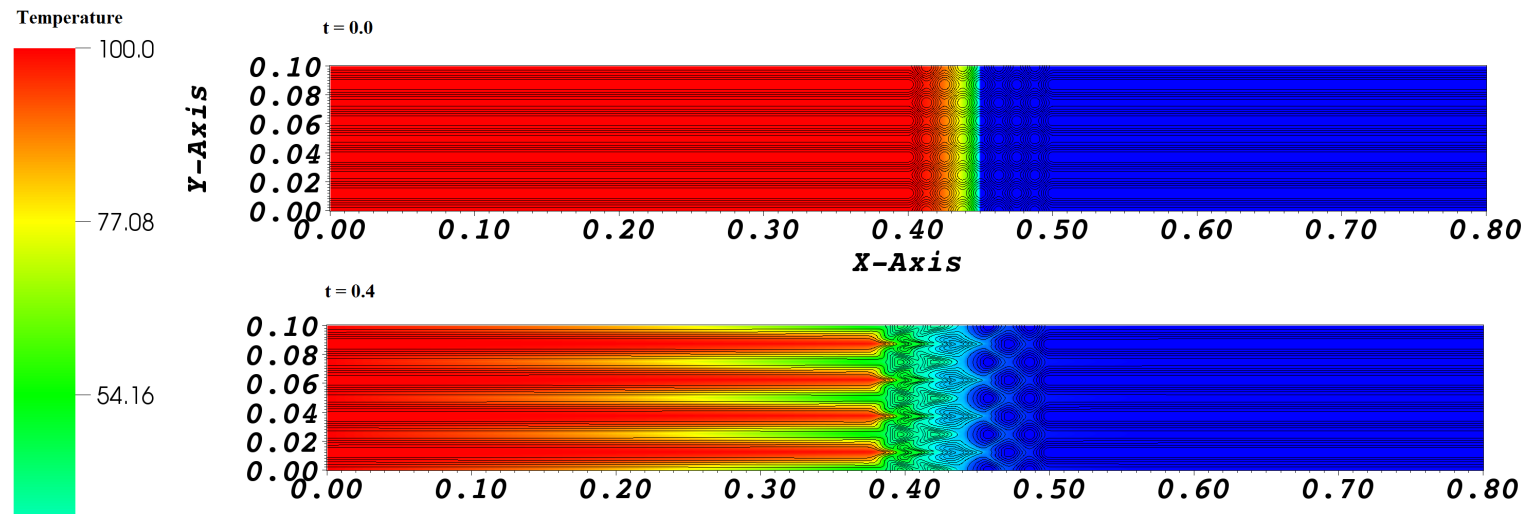

31.24

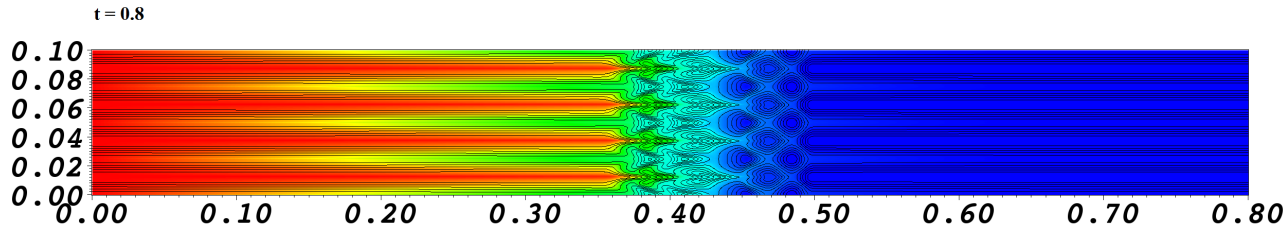

$t=1.2$

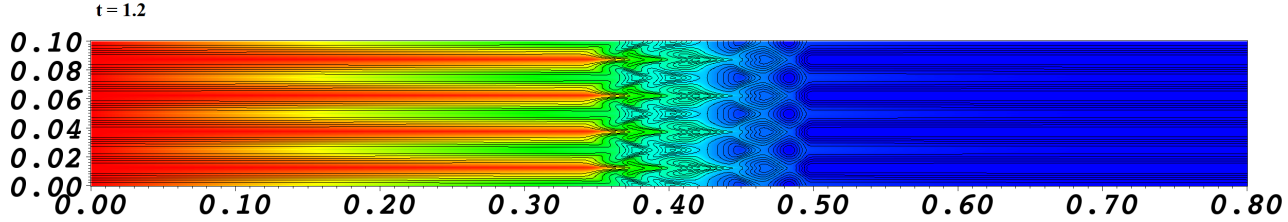

Fig. 2.- Evolution of temperature distribution with $R=0.0$. The cuts are at $(\mathrm{a}): t=0.0$, the initial state, $(\mathrm{b}): t=0.4,(\mathrm{c}): t=0.8,(\mathrm{~d}): t=1.2$, the steady state. 

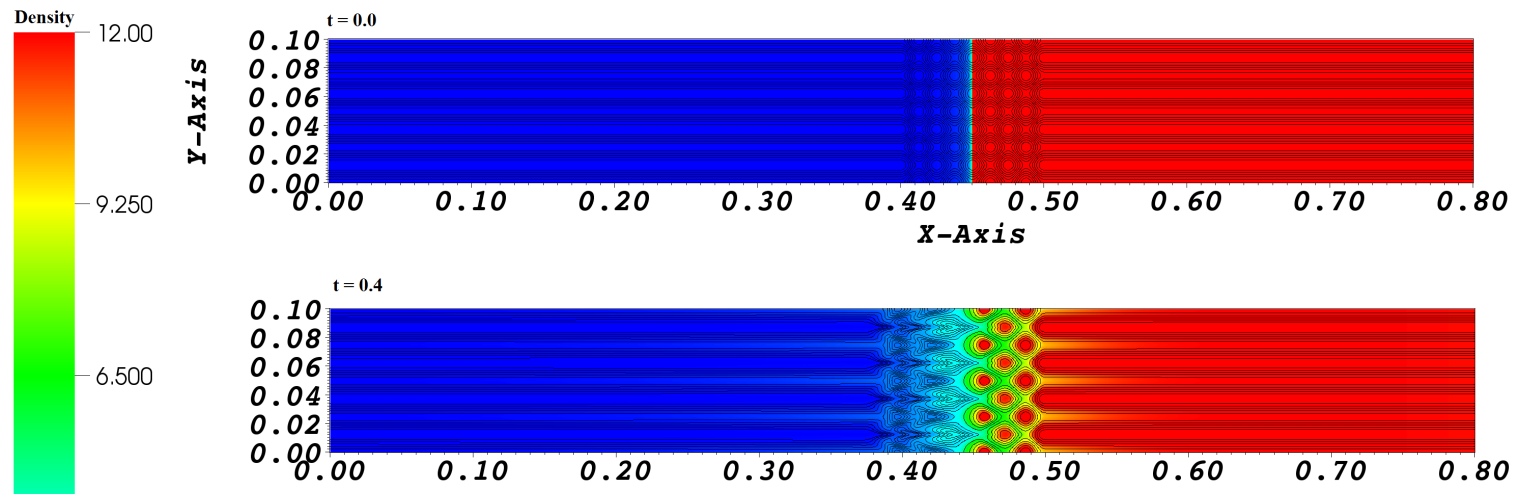

3.750
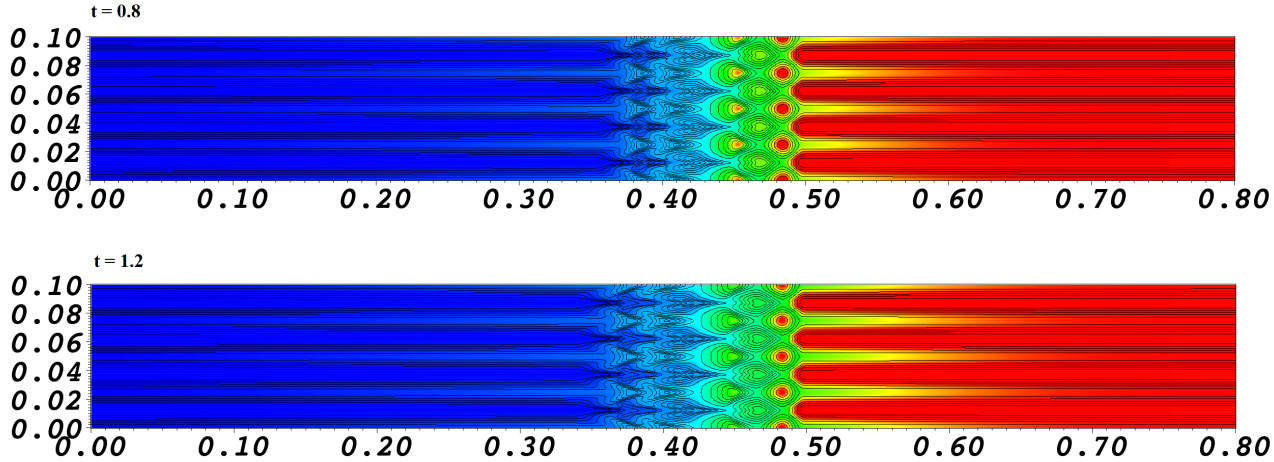

Fig. 3.- Evolution of density distribution with $R=0.0$. The cuts are at (a): $t=0.0$, the initial state, (b): $t=0.4,(\mathrm{c}): t=0.8,(\mathrm{~d}): t=1.2$, the steady state. 

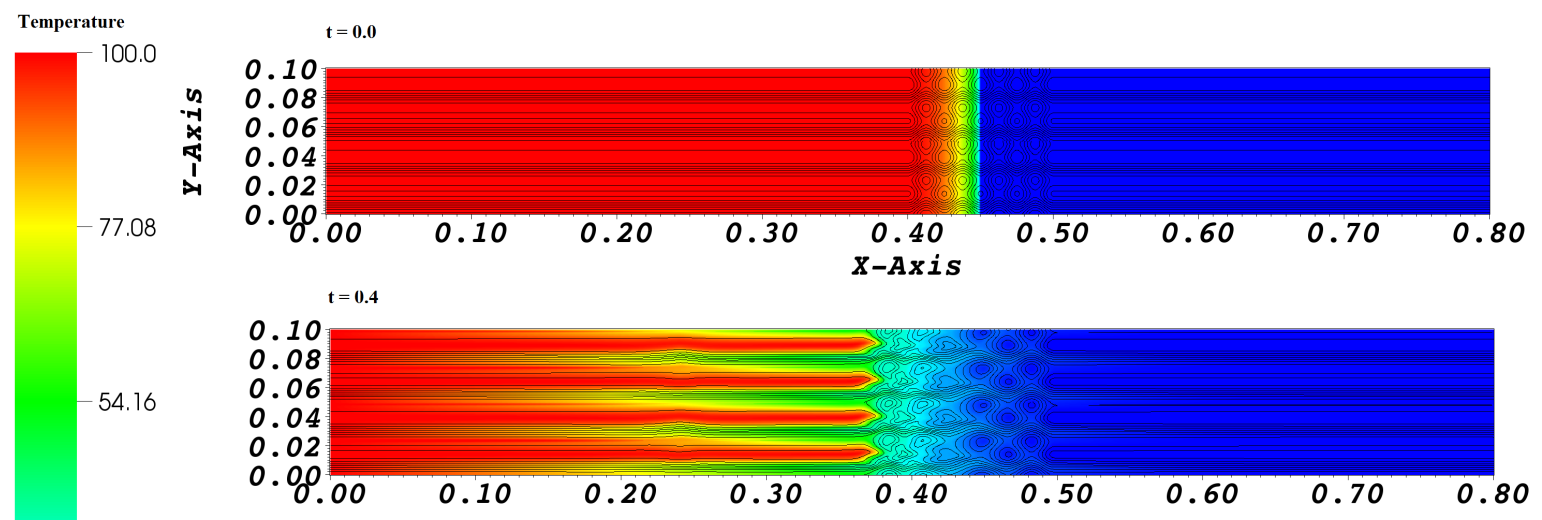

31.24
8.320

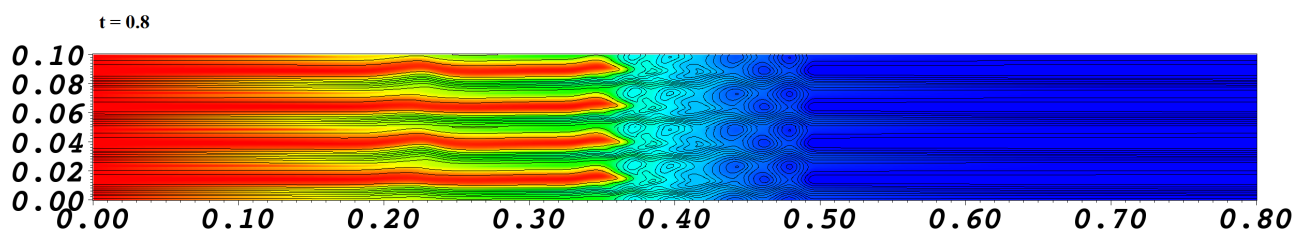

$\mathrm{t}=1.2$

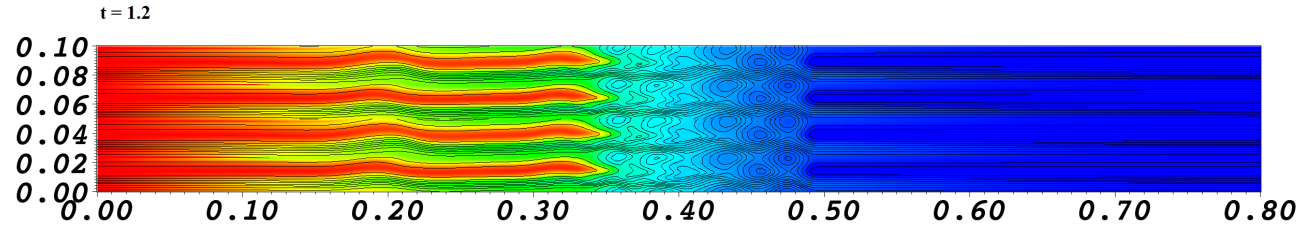

Fig. 4. - Evolution of temperature distribution with $R=0.4$. The cuts are at $(\mathrm{a}): t=0.0$, the initial state, $(\mathrm{b}): t=0.4,(\mathrm{c}): t=0.8,(\mathrm{~d}): t=1.2$, the steady state. 

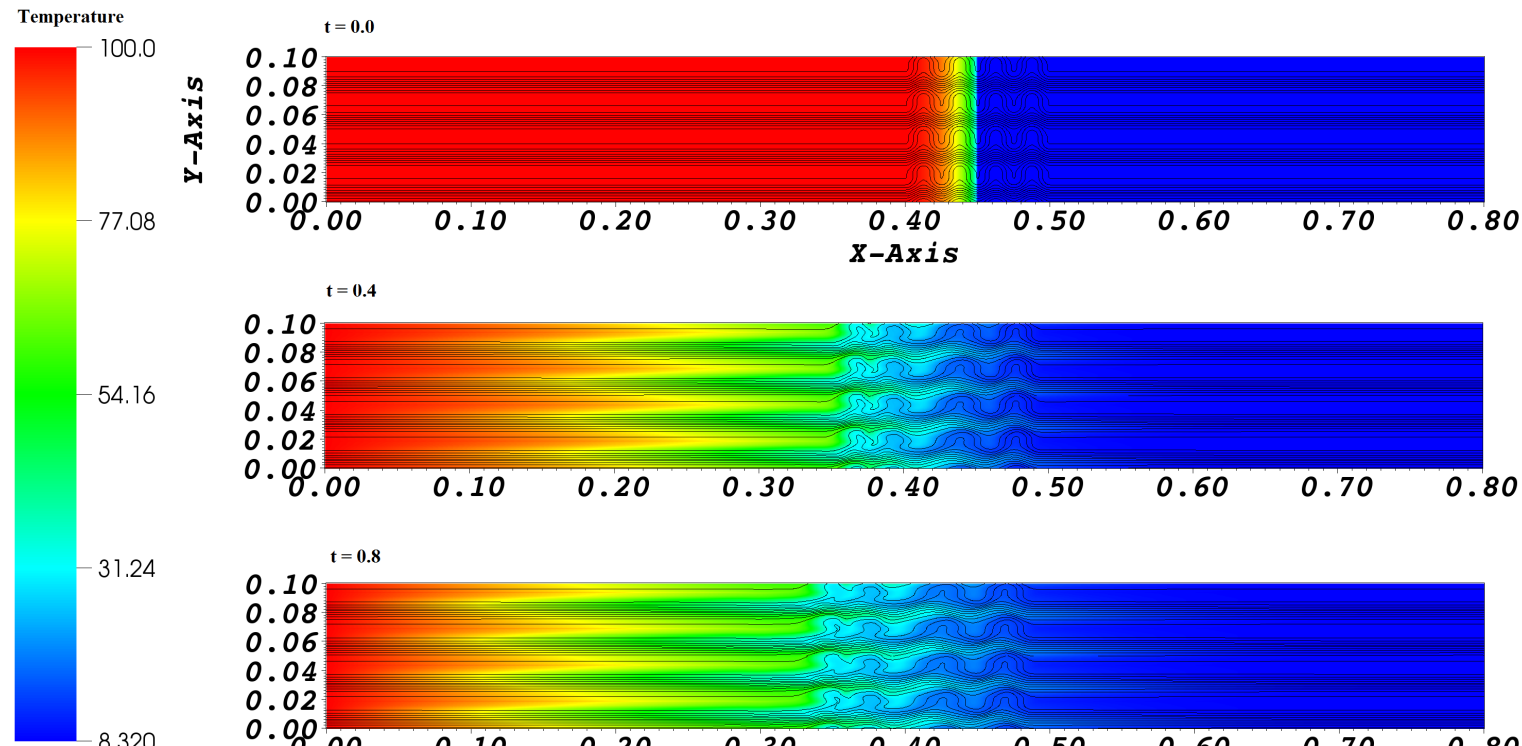

1.24
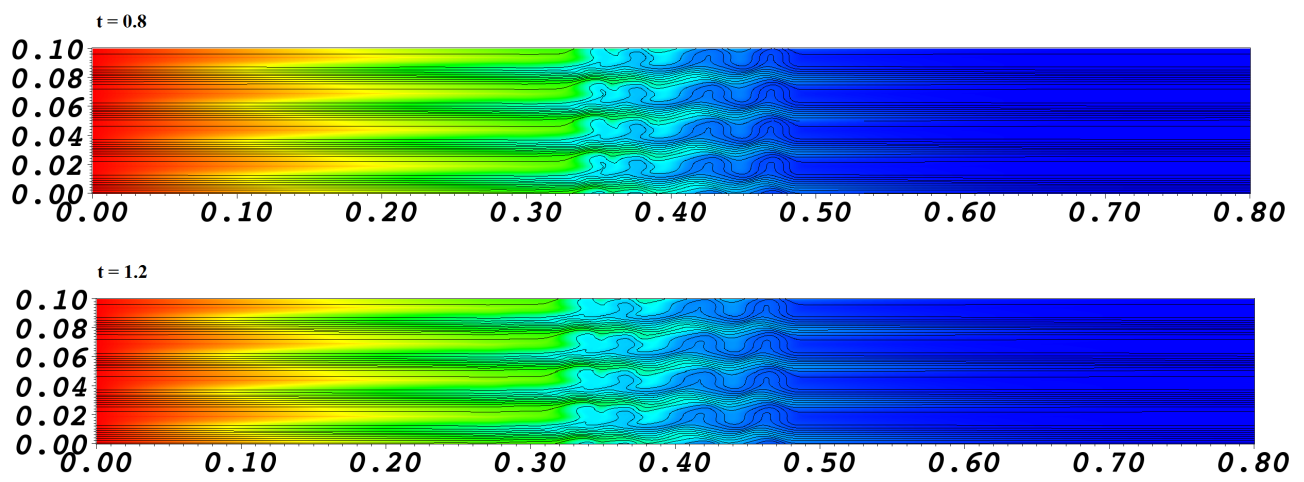

Fig. 5. - Evolution of temperature distribution with $R=1.0$. The cuts are at (a): $t=0.0$, the initial state, (b): $t=0.4,(\mathrm{c}): t=0.8,(\mathrm{~d}): t=1.2$, the steady state. 

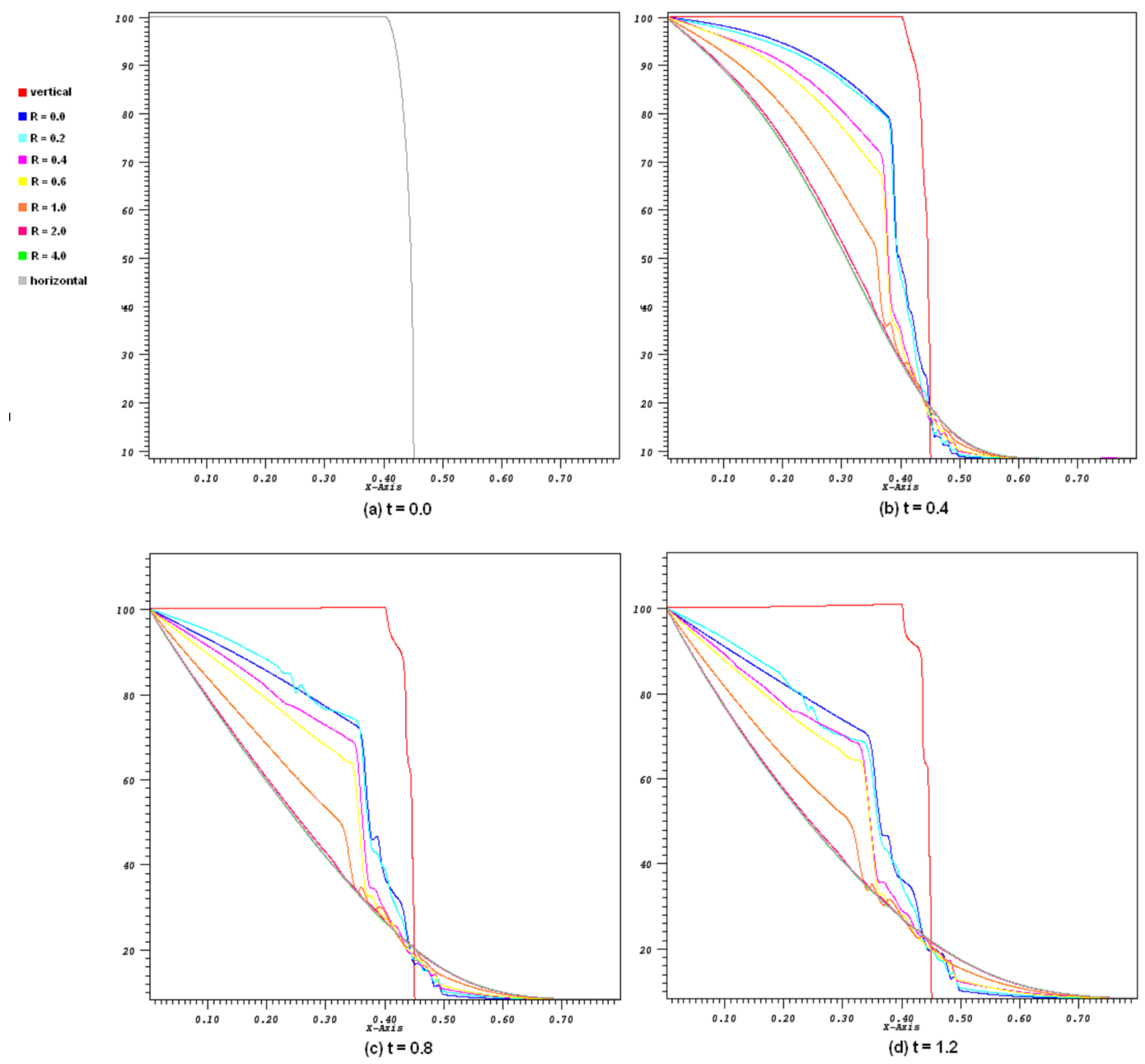

Fig. 6.- Evolution of mean cut temperature averaged on y direction with different $R$ values labeled by different colors. The cuts are at (a): $t=0.0$, the initial state, (b): $t=0.4$, (c): $t=0.8,(\mathrm{~d}): t=1.2$, the steady state. 

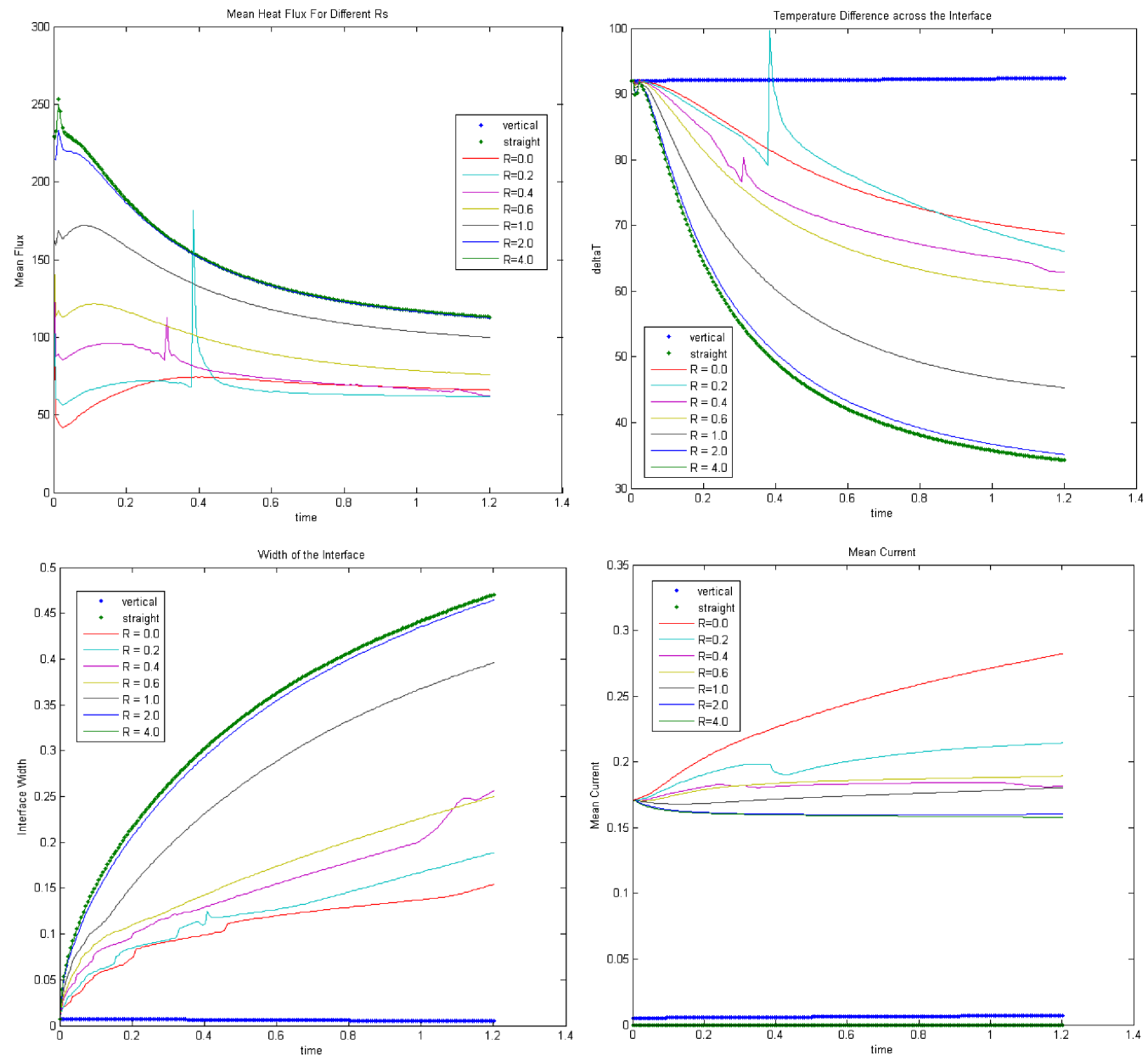

Fig. 7.- (a) top left: time evolution of mean heat flux at the interface, (b) top right: time evolution of average temperature difference between the hot and cold regions, (c) bottom left: time evolution of interface width, (d) bottom right: time evolution of the mean value of $|\nabla \times \mathbf{B}|$. 

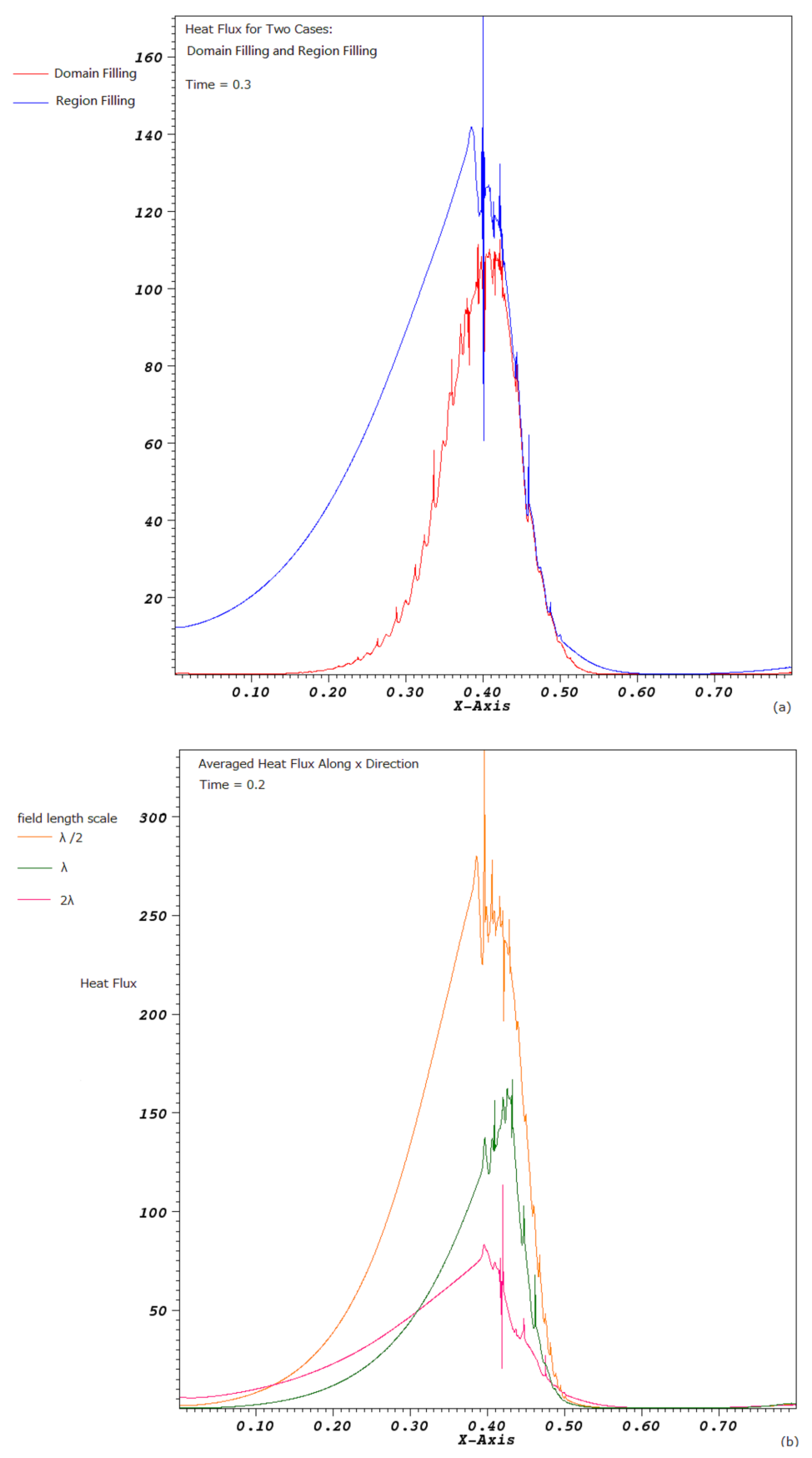

Fig. 8.- (a) Comparison of averaged heat flux for situation with field loops filling up the entire domain and situation with field loops only fill the interaction region. (b) Comparison of averaged heat flux for situations with different tangeld field length scale. 

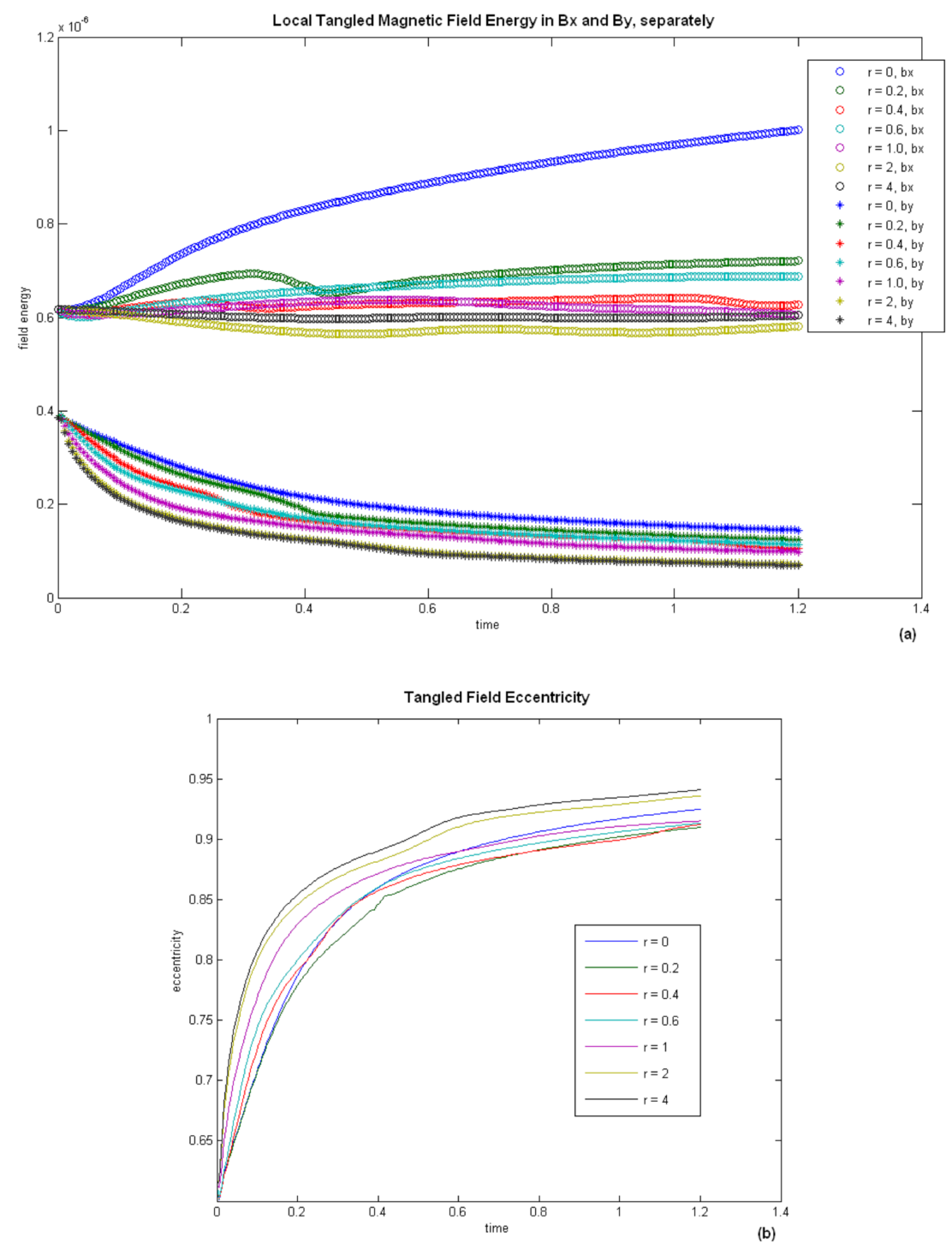

Fig. 9.- (a) Comparison on evolution of local field energy in terms of $B_{x}$ and $B_{y}$. Circles corresponds to the $B_{x}^{2} / 2$ curve, stars corresponds to the $B_{y}^{2} / 2$ curve. The different colors denote various $R$ values. (b) Eccentricity of the ellipses constructed by assigning the mean values of local $\left|B_{x}\right|$ and $\left|B_{y}\right|$ to the major and minor axes, respectively. The set of curves show different evolution patterns for different $R$ values. 


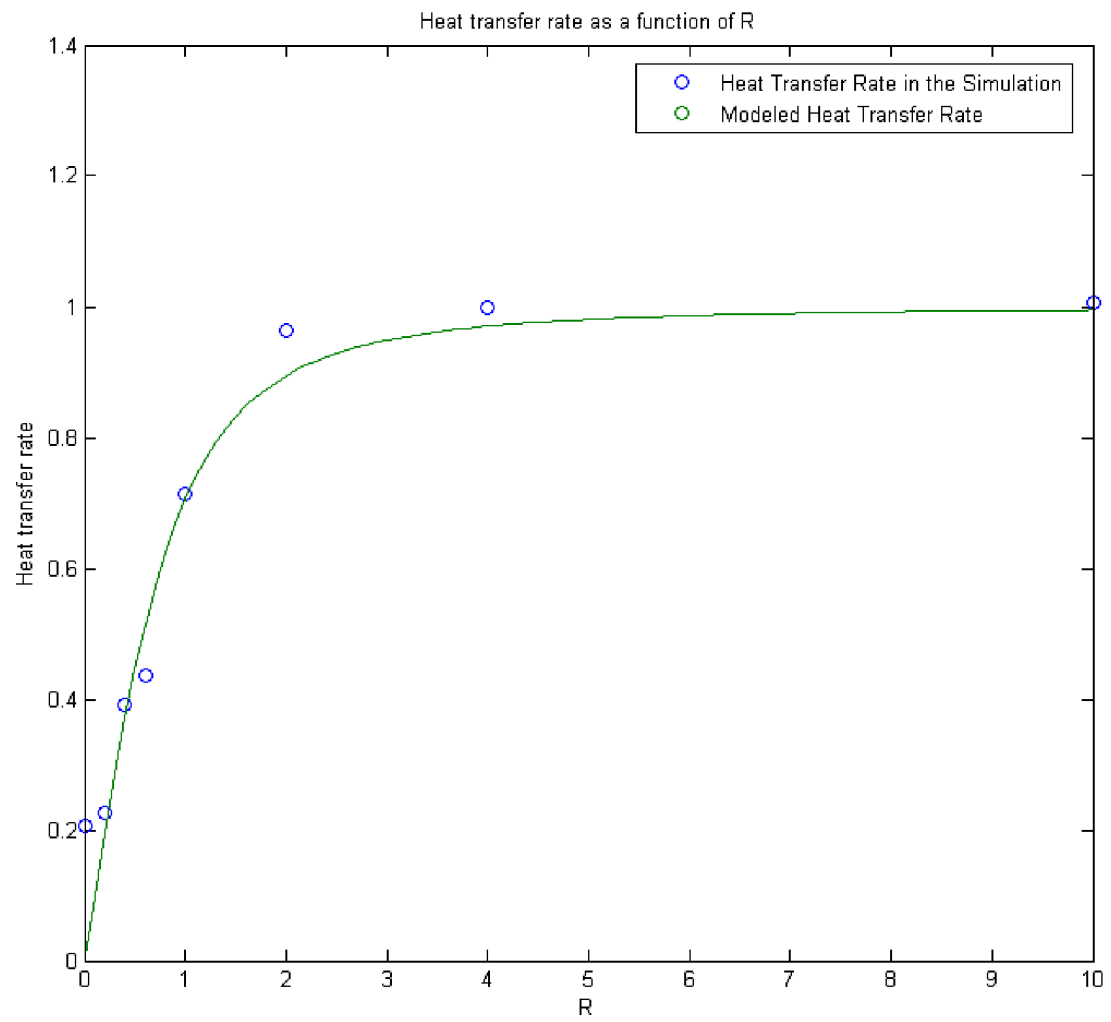

Fig. 10.- heat transfer rate observed in the simulation compared with the analytic model. 

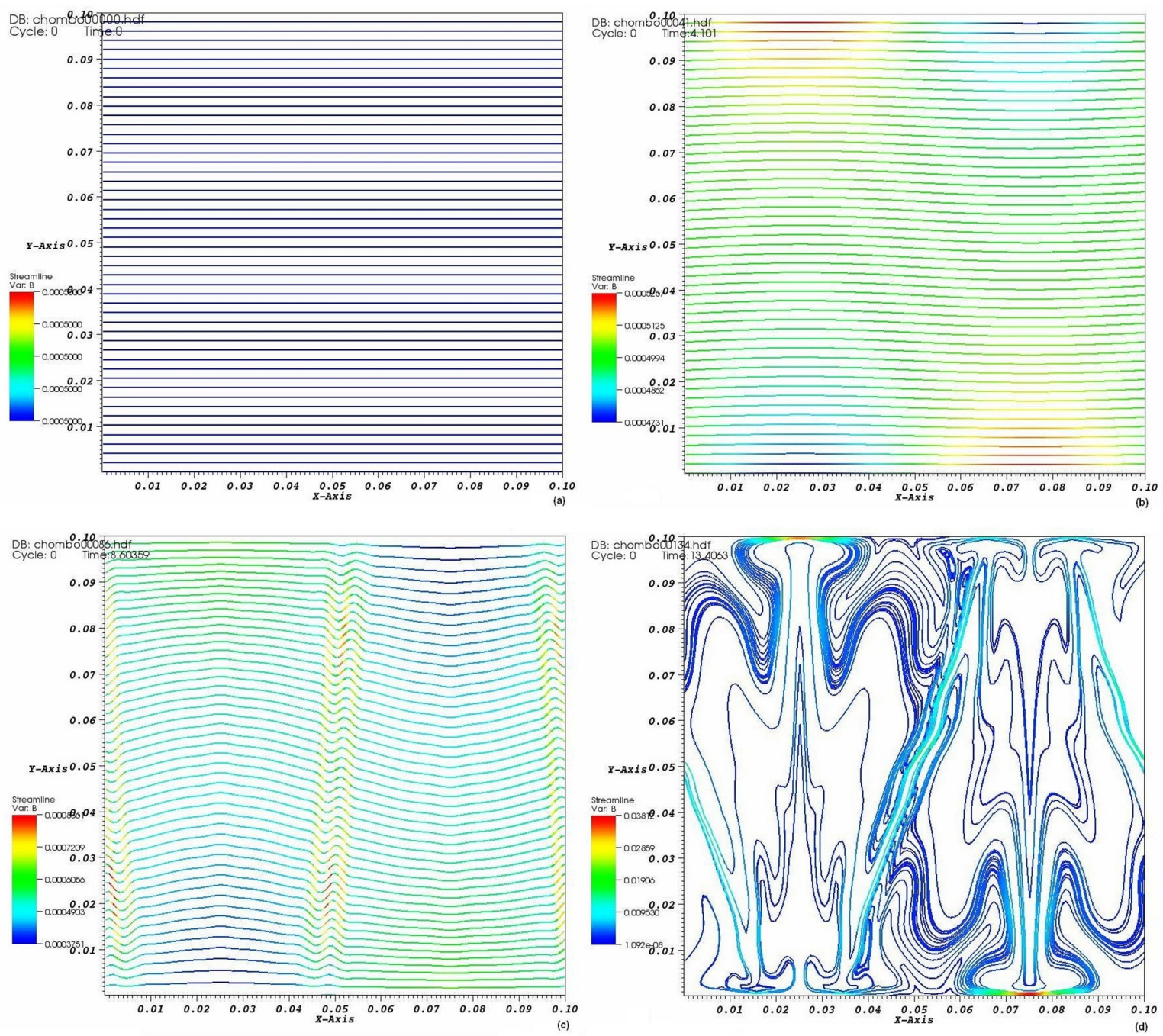

Fig. 11. - Field line evolution of magneto-thermal instability. (a): initial state. (b): $t=$ $75 \tau_{s} .(\mathrm{c}) t=150 \tau_{s} .(\mathrm{d}): t=250 \tau_{s}$. 

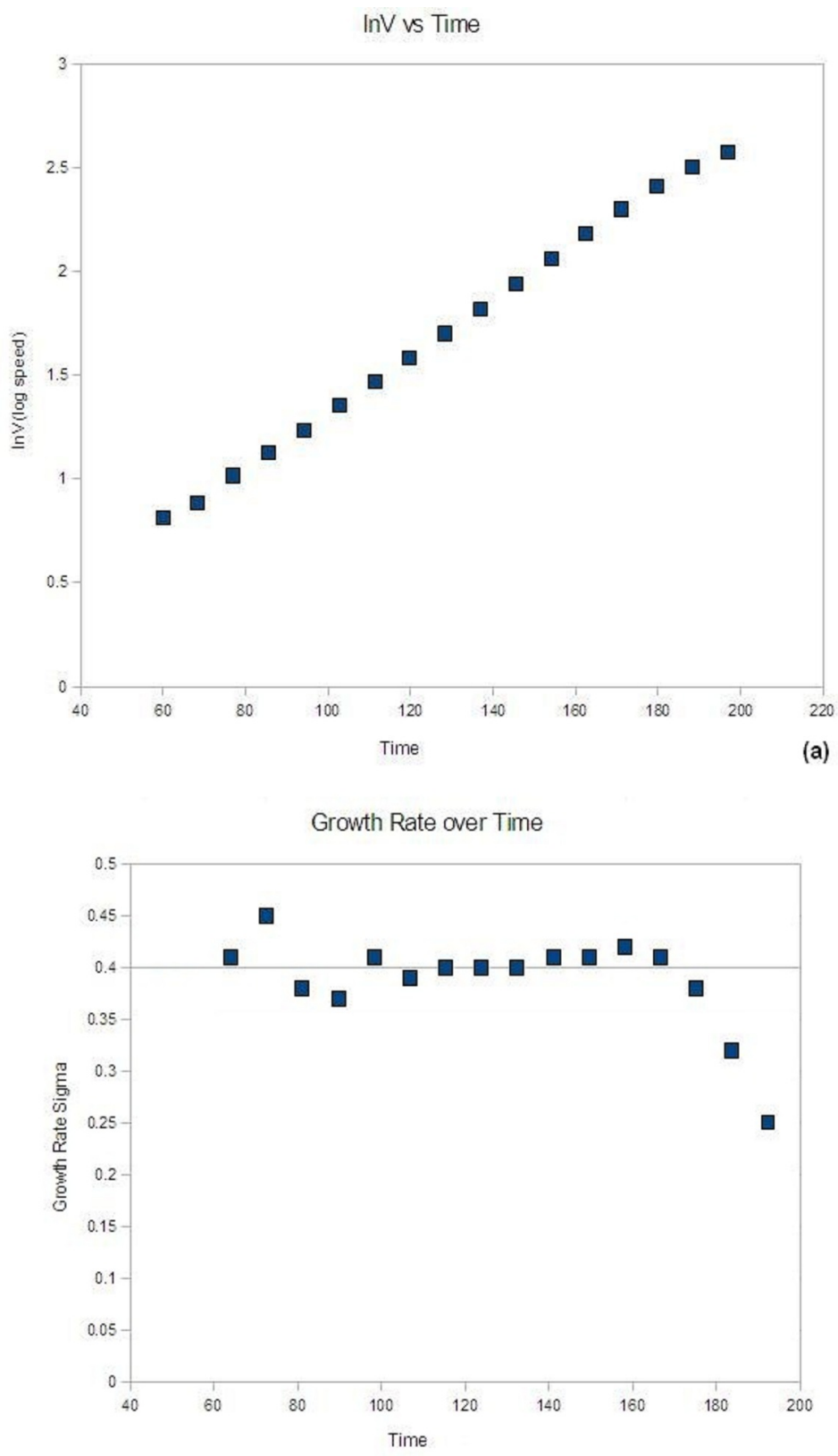

(b)

Fig. 12.- (a): ln $v_{y}$ against evolution time in $\tau_{s}$. (b): calculated growth rate against evolution time in computational units. Initially the growth rate is stable around the theoretical value 0.4 and then decreases sharply after $t=200$, which indicates the evolution has entered the nonlinear regime. 

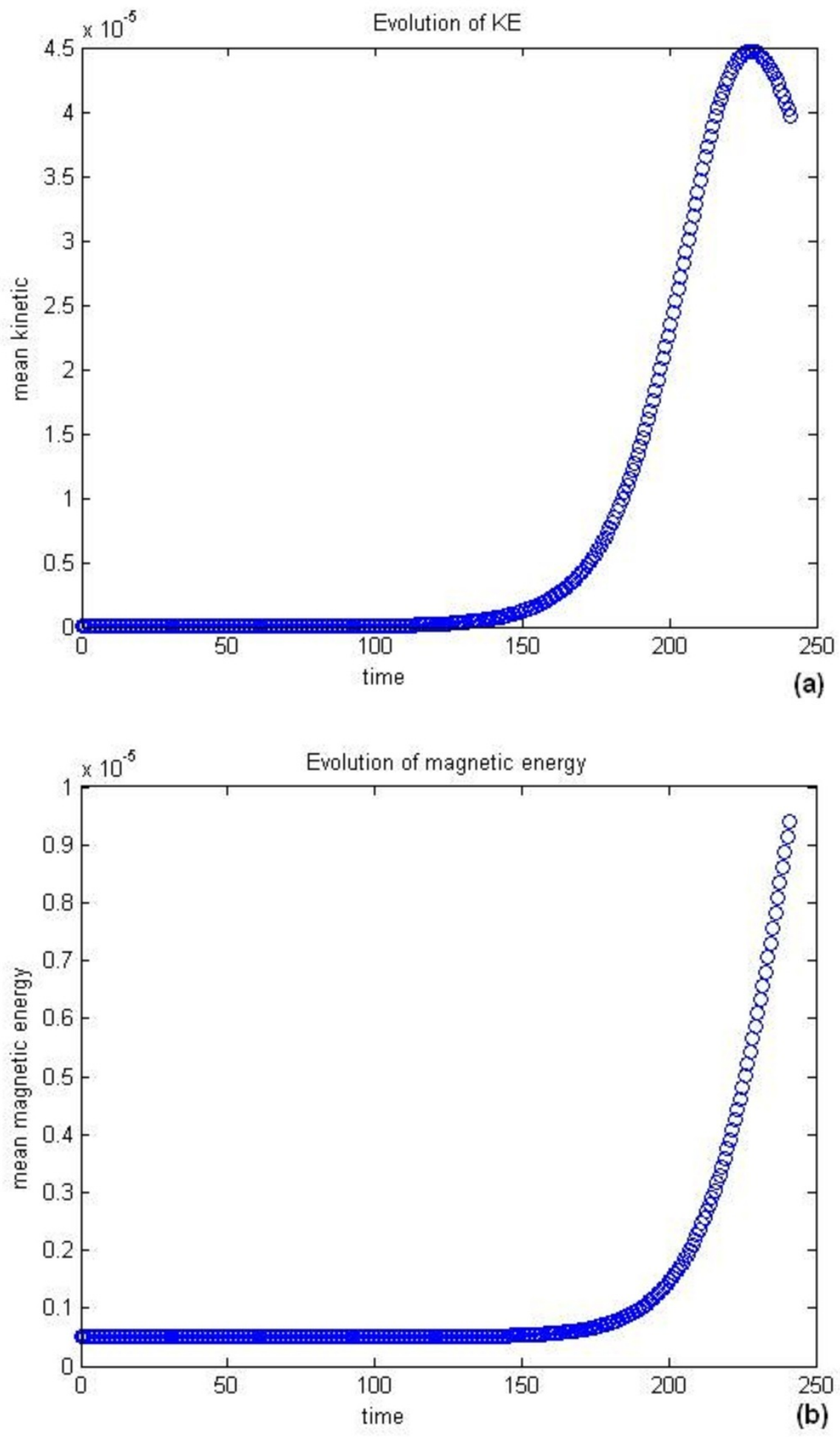

Fig. 13. - (a): evolution of mean kinetic energy. (b): evolution of mean magnetic energy. 
Table 1: Scaling of Simulation Parameters

\begin{tabular}{|c|c|c|}
\hline Variables & Computional Units & WBB \\
Number Density & 1 & $1 \mathrm{~cm}^{-3}$ \\
Temperature & 100 & $1 \mathrm{kev}$ \\
Domain Length & 0.1 & $0.025 \mathrm{pc}$ \\
Local Field Strength & $10^{-3}$ & $2^{-8}$ Gauss \\
Global Field Strength & $10^{-4}$ & $2^{-9} \mathrm{Gauss}$ \\
Evolution Time & 1.2 & $12,000 \mathrm{yrs}^{-1}$ \\
Heat Conductivity & $10^{-2}$ & $2 \times 10^{-18} \mathrm{~cm} \mathrm{~s}^{-1} \mathrm{~K}^{-2.5}$ \\
\hline
\end{tabular}

